\title{
Nueva alternativa para relleno y camuflaje de dorso nasal: Injerto mixto de L-PRF y cartílago
}

\author{
New alternative for filling and camouflage of nasal dorsum: \\ Mixed graft of L-PRF and cartilage
}

\author{
Mario Tapia C1, Alfredo Santamaría C1.
}

\begin{abstract}
RESUMEN
Introducción: Entre las alteraciones del dorso nasal puede encontrarse desde un rádix bajo hasta un aplastamiento severo del dorso nasal, siendo fundamental el uso de injertos y material de relleno.

Objetivo: Proponer una nueva alternativa de relleno y camuflaje en rinoplastía mediante un injerto mixto de L-PRF más cartílago picado en una matriz de fibrina autóloga.

Material y método: Evaluación de los pacientes operados de rinoseptoplastía en quienes se utilizó el injerto mixto entre febrero y septiembre del año 2016 en el Servicio de Otorrinolaringología del Hospital Regional de Concepción. Evaluación de resultados respecto a reabsorción e irregularidades del injerto y autoevaluación del grado de satisfacción.

Resultados: Fueron operados 7 pacientes. Cuatro de ellos presentaban aplastamiento severo de dorso nasal y tres un rádix profundo. Se logró el relleno y camuflaje del área deseada en todos los casos. No se presentó desplazamiento ni reabsorción del injerto en ninguno de los casos. El uso de este injerto no significó mayor costo económico, y el grado de satisfacción fue alto en todos los casos.

Conclusión: Primer estudio en utilizar un injerto mixto de cartílago más L-PRF en una matriz de fibrina autóloga en rinoplastía, demostrando ser una alternativa asequible, efectiva, reproducible y segura, particularmente útil en casos de revisión.
\end{abstract}

Palabras clave: Rinoplastía, fibrina rica en plaquetas y leucocitos, aumento de dorso nasal, injerto autólogo.

\section{ABSTRACT}

Introduction: Among the alterations of the nasal dorsum there can be found from a low radix to a severe crushing of the nasal dorsum, being fundamental the use of grafts and filling materials.

Aim: To propose a new filling and camouflage alternative in rhinoplasty by means of a mixed graft of L-PRF and chopped cartilage in an autologous fibrin matrix.

Médicos del Servicio de Otorrinolaringología y Cirugía de Cabeza y Cuello, Hospital Guillermo Grant Benavente, Concepción, Chile. 
Material and method: Evaluation of the operated patients of rhinoseptoplasty in whom the mixed graft was used between February and September 2016 in the Otorhinolaryngology unit of the Hospital Regional de Concepción. Evaluation of results regarding resorption and graft irregularities and self-assessment of degree of satisfaction.

Results: Seven patients were operated. Four of them presented severe crushing of the nasal dorsum and three of them a deep radix. The filling and camouflage of desired area was achieved in all cases. There were no displacement or resorption of the graft in any case. The use of this graft did not mean greater economic cost, and the degree of satisfaction was high in all cases.

Conclusions: First study to use a mixed graft of cartilage and L-PRF in an autologous fibrin matrix in rhinoplasty, proving to be an affordable, effective, reproducible and safe alternative, particularly useful in revision surgeries.

Key words: Rhinoplasty, platelet-rich fibrin and leukocytes, nasal dorsal augmentation, autologous graft.

\section{INTRODUCCIÓN}

Entre las alteraciones a tratar en rinoplastía funcional y estética, los pacientes con dorso nasal cóncavo, rádix profundo, dorso en silla de montar 0 incluso aplastamiento nasal, representan un desafío. Estas pueden corresponder a variables anatómicas 0 a secuelas postraumáticas donde reconstruir la forma y la altura del dorso con un contorno sin irregularidades constituye el principal objetivo.

Para la corrección de este tipo de alteraciones es importante el relleno a utilizar existiendo una amplia variedad de injertos. En este contexto es importante comprender las propiedades e interacción del injerto utilizado con el tejido subyacente, ya que éstos pueden influir en los resultados a mediano y largo plazo.

El injerto de cartílago septal es la alternativa más utilizada para aumentar el dorso nasal y sus beneficios han sido ampliamente descritos ${ }^{1,2}$. Cuando el cartílago septal resulta insuficiente, el cartílago auricular representa otra alternativa, sin embargo, debido al tipo de cartílago y sus características puede no ser aplicable cuando sea necesario realizar grandes aumentos ${ }^{3}$. Cuando no es posible obtener un injerto adecuado con los anteriormente descritos, el cartílago costal representa una buena alternativa ${ }^{4}$, no obstante, se describe mayor probabilidad de presentar deformaciones del injerto con el tiempo que pueden generar irregularidades palpables e incluso visibles asociado a la mayor complejidad y morbilidad que conlleva la obtención de éste.

En los últimos 15 años han sido descritos métodos que utilizan cartílago cortado en pequeños fragmentos como relleno para aumento del dorso nasal, describiéndose una serie de beneficios entre los cuales destaca la posibilidad de darle forma y moldearlos manualmente ${ }^{5,6}$.

Los concentrados autólogos ricos en plaquetas y fibrina han sido utilizados durante años para promover la cicatrización del sitio quirúrgico ${ }^{7-9}$. Con el tiempo el enfoque de la investigación se ha desplazado hacia las propiedades curativas de las plaquetas concentradas en una matriz de fibrina y el valor de los factores de crecimiento circulantes y leucocitos se ha hecho evidente ${ }^{8-10}$. Es actualmente aceptado que todos estos elementos juegan un rol significativo en el proceso curativo, por lo tanto, en los preparados modernos ricos en plaquetas se encuentran todos estos componentes ${ }^{11}$.

La clasificación actual de estos concentrados se basa en su arquitectura de fibrina y contenido celular. Está compuesto por 2 grupos principales, el plasma rico en plaquetas (PRP) y la fibrina rica en plaquetas (PRF), las cuales están disponibles en una forma pura 0 enriquecida con leucocitos (L-PRP y L-PRF) ${ }^{12}$. Cada preparado tiene un perfil biológico único que dicta sus aplicaciones clínicas.

Los concentrados de L-PRF proporcionan una liberación prolongada de factores de crecimiento y se pueden preparar fácilmente durante la cirugía ${ }^{13-16}$. Son económicas y autólogas, por lo tanto 
evitan complicaciones como reacción a cuerpo extraño. Estos productos existen en forma de gel y debido a su matriz de fibrina de alta densidad pueden ser manejados como material sólido para otras aplicaciones.

Las técnicas de relleno a utilizar dependen de cada arquitectura nasal y éstas pueden ir desde injertos en rádix, hasta injertos parciales o totales de dorso ${ }^{17}$. En este estudio presentamos el uso de un injerto mixto compuesto por pequeñas piezas de cartílago y membranas de L-PRF en una matriz de fibrina de alta densidad obtenida de sangre periférica utilizando el proceso descrito por Choukroun ${ }^{18}$.

\section{OBJETIVO}

Nuestro principal objetivo es demostrar su efectividad como material de relleno y proponerlo como una alternativa, particularmente cuando la cantidad de injerto disponible es insuficiente.

\section{MATERIAL Y MÉTODO}

Se evaluaron todos los pacientes operados de rinoseptoplastías primarias con rádix profundos y/0 aplastamiento nasal, en quienes utilizar un injerto como relleno era necesario, entre febrero y septiembre del año 2016 en el Servicio de Otorrinolaringología y Cirugía de Cabeza y Cuello del Hospital Clínico Regional de Concepción. Se obtuvo el consentimiento informado de todos los pacientes quienes fueron previamente informados del objetivo del trabajo. Se realizó una evaluación clínica preoperatoria y posoperatoria con registro fotográfico. Para la evaluación de resultados se realizaron dos métodos, ambos con un mínimo de 6 meses posoperatorio. El primero consistió en una evaluación clínica realizada por 3 otorrinolaringólogos no involucrados en el procedimiento quirúrgico, calificando los resultados mediante una escala de Likert utilizando como variables la reabsorción del injerto y la presencia de irregularidades de dorso las cuales fueron categorizadas como; "muy bueno", "bueno", "regular", "malo" y "muy malo" (Figura 1).

El segundo método fue realizado mediante una autoevaluación por parte de los pacientes de acuerdo al grado de satisfacción con los resultados, utilizando el mismo sistema de calificación con 5 variables; "muy satisfactorio", "satisfactorio", "regularmente satisfactorio", "descontento" y "muy descontento" (Figura 2).

\section{Técnica de cartílago picado y L-PRF}

Una vez obtenido el injerto de cartílago (septal de elección), se define la cantidad necesaria para realizar el resto de los injertos en la cirugía y el que se utilizará como relleno del dorso o rádix. Cuando éste sea insuficiente para lograr el relleno adecuado se obtendrá injerto de cartílago auricular o costal. Se confecciona un molde a partir de una jeringa de 3 milímetros $(\mathrm{mm})$ la cual es cortada longitudinal y transversalmente de acuerdo con el tamaño requerido del injerto mixto, donde posteriormente se colocarán los materiales que lo componen. El

\begin{tabular}{|l|l|l|l|l|l|}
\hline & Muy bueno & Bueno & Regular & Malo & Muy malo \\
\hline $\begin{array}{l}\text { Reabsorción } \\
\text { Irregularidades }\end{array}$ & & & & & \\
\hline
\end{tabular}

Figura 1. Escala de evaluación de resultados.

\begin{tabular}{|l|l|l|l|l|l|}
\hline & Muy satisfactorio & Satisfactorio & Regularmente satisfactorio & Descontento & Muy descontento \\
\hline $\begin{array}{l}\text { Grado de } \\
\text { satisfacción }\end{array}$ & & & & & \\
\hline
\end{tabular}

Figura 2. Escala de autoevaluación de resultados. 
primer paso corresponde a cortar el cartílago en pequeñas piezas menores a 1-2 $\mathrm{mm}$ con un bisturí, estos trocitos no deben ser machacados ni aplastados, y posteriormente se colocan en el molde fabricado mientras se preparan las láminas de L-PRF (Figura 3).

La membrana de L-PRF se obtiene de manera similar a la técnica descrita por Chouckroun ${ }^{18}$. Se extrae sangre periférica de una vía arterial o venosa en tubos de 10 centímetros cúbicos (cc) libre de anticoagulante, los que posteriormente son centrifugados a 2.700 revoluciones por minuto (rpm) durante 12 minutos. Una vez centrifugados se extrae el coágulo de fibrina desde el tubo con una pinza y se coloca en la caja de preparación ejerciendo compresión de las membranas con la tapa de ésta obteniéndose finalmente membranas de 1 a $2 \mathrm{~mm}$

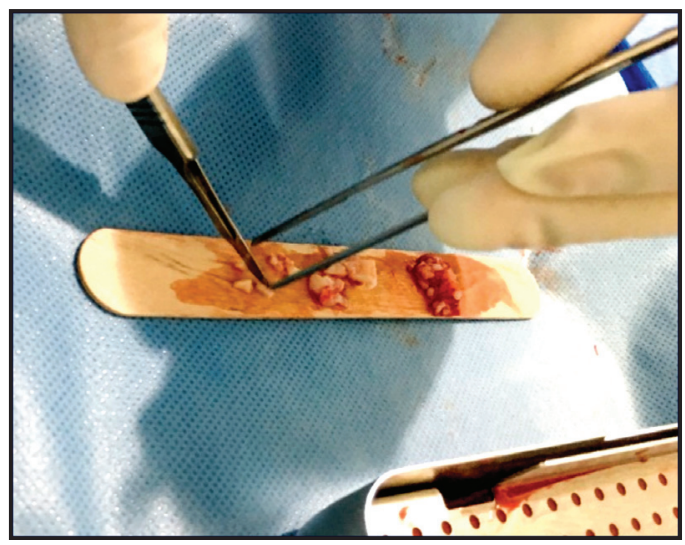

Figura 3. Cartílago septal fragmentado en pequeñas piezas.

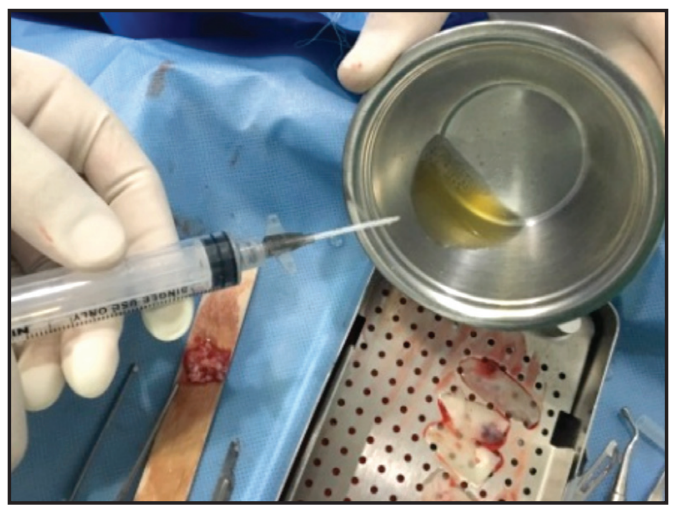

Figura 5. Obtención de fibrinógeno. de grosor. Finalmente, ésta es cortada en pequeños fragmentos de igual tamaño que los de cartílago y mezclado con éstos en el molde (Figura 4).

Estos injertos conforman una mezcla heterogénea con los fragmentos libres entre sí, razón por la cual se diseñó un tercer paso para constituir un injerto compuesto estable.

El tercer componente y último paso es similar al segundo, no obstante, esta vez la sangre obtenida se centrifuga a $2.700 \mathrm{rpm}$ durante sólo 3 minutos, obteniéndose un concentrado líquido de fibrinógeno. Este concentrado debe mezclarse en menos de 2 minutos con los otros componentes dispuestos en el molde diseñado previamente obteniéndose el injerto mixto, actuando este último centrifugado como un pegamento autólogo (Figuras 5 y 6 ).

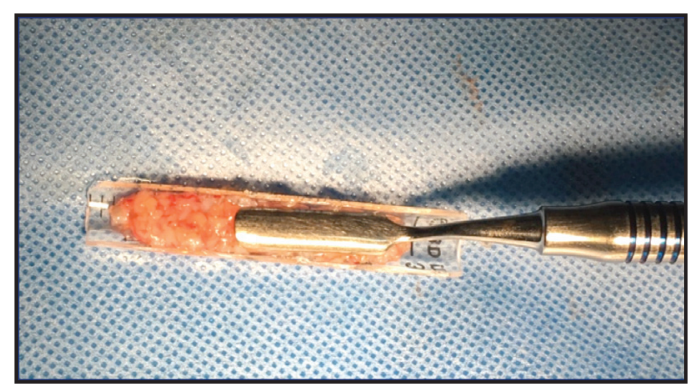

Figura 4. Molde de injerto con pequeños fragmentos de cartílago y láminas de L-PRF.

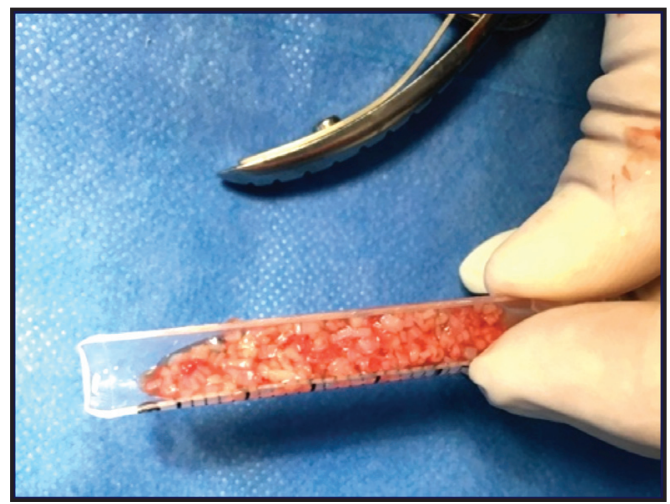

Figura 6. Molde con injerto autólogo mixto de cartílago y láminas de L-PRF en una matriz de fibrinógeno. 


\section{Colocación del injerto y modelado en dorso nasal}

Colocamos nuestro injerto sobre la superficie del dorso nasal o rádix con la ayuda de un retractor Aufricht y una pinza (Figura 7).

Una vez posicionado es posible modelar el injerto digitalmente para optimizar detalles. No realizamos sobrecorrección en ninguno de los pacientes intervenidos. Una vez finalizada la cirugía se posicionan férulas de Doyle y férula nasal externa con tela adhesiva sobre la piel y yeso sobre ésta.

\section{RESULTADOS}

Todos los pacientes fueron operados por el mismo cirujano mediante abordaje abierto con anestesia general. En el periodo descrito se incluyeron 7 pacientes. 2 pacientes masculinos y 5 pacientes femeninos, con un rango de edad entre 18 a 59 años (un promedio de 37,1). Con un intervalo de seguimiento de 17 a 24 meses (promedio de 20,1 meses). De éstos, 4 pacientes presentaban un aplastamiento nasal severo todos secundarios a secuela traumática, en quienes se realizó un injerto total de dorso nasal (Figuras 8 y 9 ).

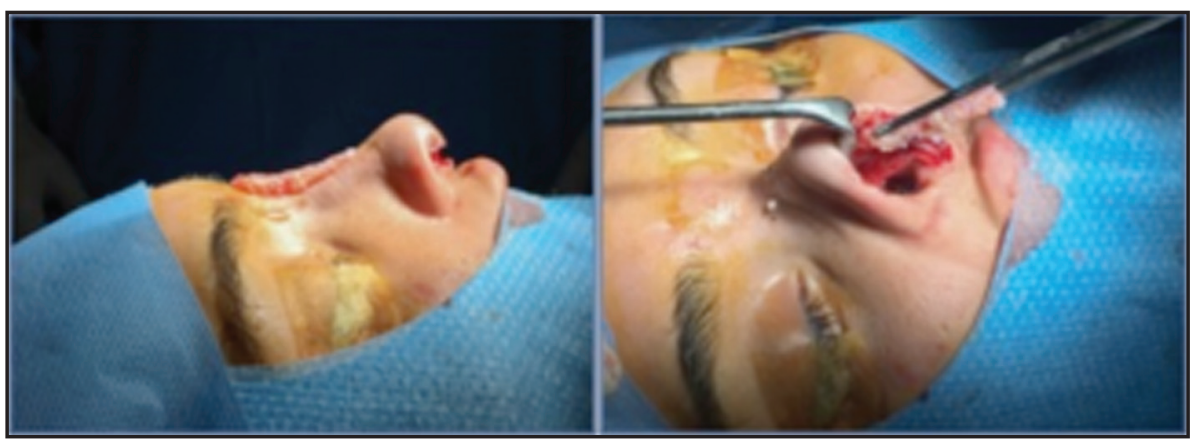

Figura 7. Posicionamiento de injerto mixto en dorso nasal.

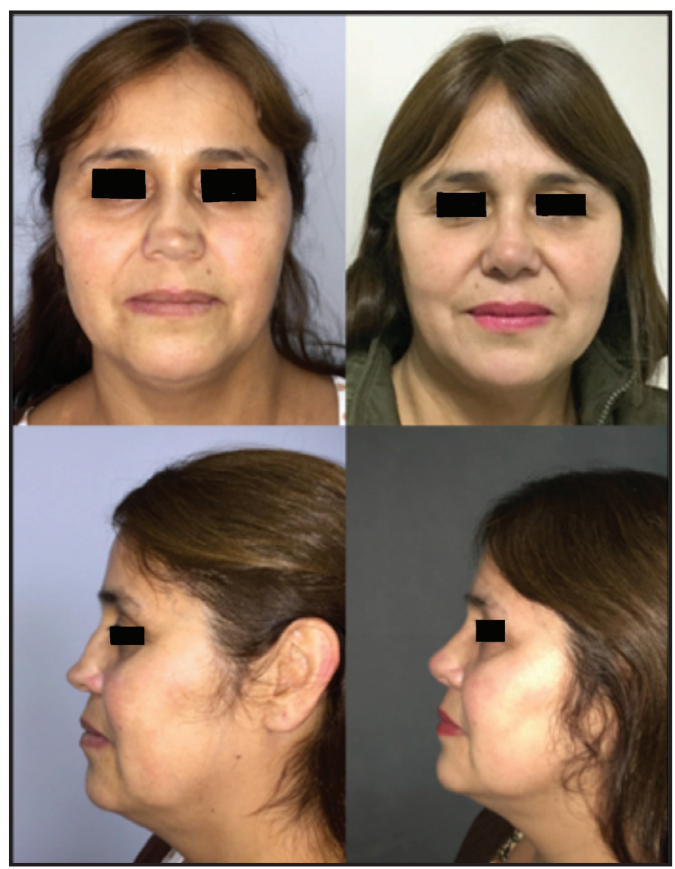

Figura 8. Injerto mixto de dorso en un caso de aplastamiento nasal severo.

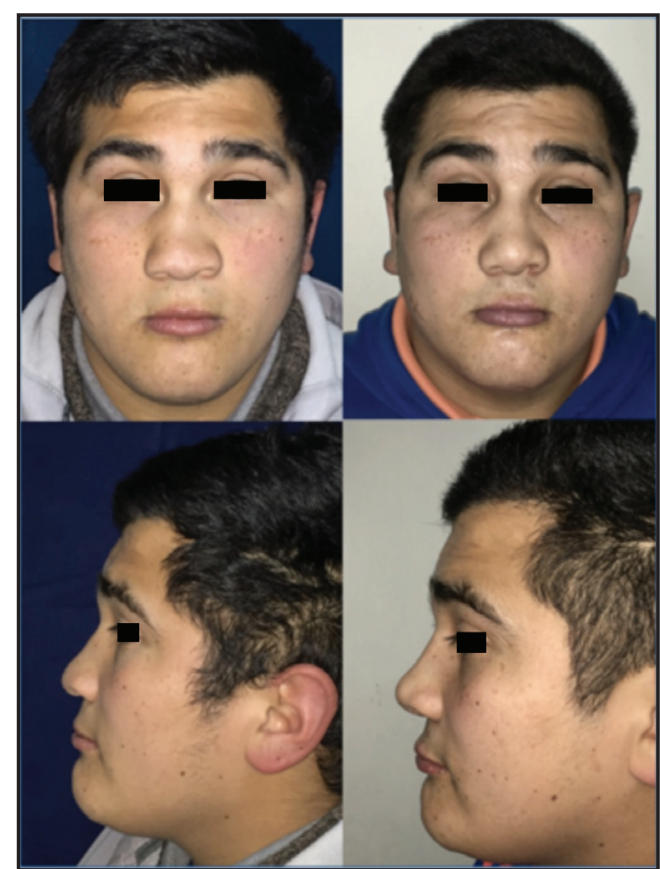

Figura 9. Injerto mixto de dorso en un caso de aplastamiento nasal severo. 
Los restantes tres pacientes presentaban un rádix profundo, en quienes se posicionó injertos de media longitud de dorso o solo como relleno de rádix (Figuras 10 y 11).

Respecto a la reabsorción, los otorrinolaringólogos evaluadores externos calificaron como resultado "Muy bueno" a 5 de los 7 casos. Si bien en ninguno de los casos se presentó reabsorción del injerto hasta la fecha, el volumen obtenido en los 2 primeros pacientes operados no fue considerado óptimo por los autores, lo que se atribuye a la estimación inexacta de la cantidad requerida de relleno al momento de la cirugía. Respecto a las irregularidades se calificó como "Muy bueno" a 4 casos, "Bueno" 2 casos y "Regular" 1 caso, sin casos calificados como malos o muy malos (Tabla 1).

Los resultados menos favorables fueron registrados para los primeros 3 pacientes operados, resultando importante mencionar que en aquellos pacientes se utilizó un molde a partir de una jeringa de $5 \mathrm{cc}$, que posteriormente fue modificada por una de $3 \mathrm{cc}$. Hasta la fecha no se ha requerido realizar ninguna reintervención.

Como resultados de la autoevaluación de acuerdo al grado de satisfacción; 5 pacientes calificaron sus resultados como "Muy satisfactorio", y 2 pacientes como "Satisfactorio", sin casos calificados como "regularmente satisfactorio", "descontentos" o "muy descontentos" con los resultados obtenidos (Tabla 2).

\section{DISCUSIÓN}

Los beneficios del uso de cartílago cortado en pequeños fragmentos como injerto de relleno de dorso nasal han sido descritos por varios autores ${ }^{5,6}$. La posibilidad de confeccionar la forma requerida y moldearlo una vez posicionado es uno de sus beneficios más destacables. Al comienzo, parecía necesario el uso de una envoltura con el fin de prevenir el desplazamiento de pequeños fragmentos de cartílago, disminuir la probabilidad de irregularidades y de reabsorción.

Erol ${ }^{19}$ reportó una extensa serie de casos con una técnica que denominó "delicia turca", la cual consistía en envolver cartílago cortado en pequeñas piezas de 0,5 a $1 \mathrm{~mm}$ en celulosa regenerada oxidada $\left(\right.$ Surgice ${ }^{\circledR}$ ) con una forma cilíndrica. Las

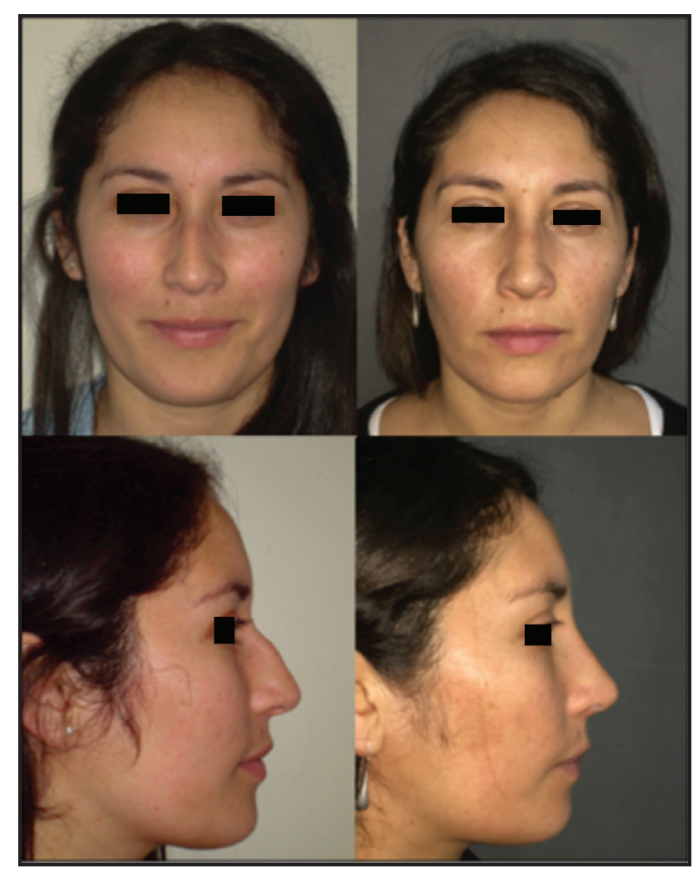

Figura 10. Injerto mixto como relleno en un caso de rádix profundo.

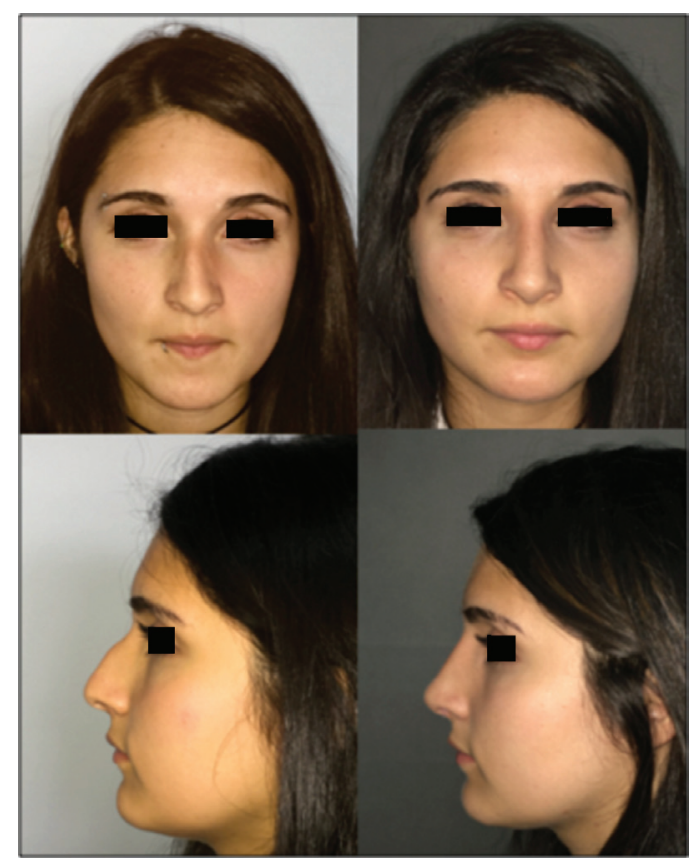

Figura 11. Injerto mixto de media longitud de dorso.

muestras histológicas de sus injertos evidenciaron la presencia de islas de tejido cartilaginoso rodeado de fibrosis. Elahi y cols ${ }^{20}$ utilizaron injertos de 
Tabla 1. Resultado registrado por evaluadores externos

\begin{tabular}{|c|c|c|c|c|}
\hline & & Muy bueno & Bueno & Regular \\
\hline \multirow[t]{2}{*}{ Caso 1} & Reabsorción & & $\checkmark$ & \\
\hline & Irregularidades & & $\checkmark$ & \\
\hline \multirow[t]{2}{*}{ Caso 2} & Reabsorción & & $\checkmark$ & \\
\hline & Irregularidades & & & $\checkmark$ \\
\hline \multirow[t]{2}{*}{ Caso 3} & Reabsorción & $\checkmark$ & & \\
\hline & Irregularidades & & $\checkmark$ & \\
\hline \multirow[t]{2}{*}{ Caso 4} & Reabsorción & $\checkmark$ & & \\
\hline & Irregularidades & $\checkmark$ & & \\
\hline \multirow[t]{2}{*}{ Caso 5} & Reabsorción & $\checkmark$ & & \\
\hline & Irregularidades & $\checkmark$ & & \\
\hline \multirow[t]{2}{*}{ Caso 6} & Reabsorción & $\checkmark$ & & \\
\hline & Irregularidades & $\checkmark$ & & \\
\hline \multirow[t]{2}{*}{ Caso 7} & Reabsorción & $\checkmark$ & & \\
\hline & Irregularidades & $\checkmark$ & & \\
\hline
\end{tabular}

Tabla 2. Resultados de autoevaluación

\begin{tabular}{|l|c|c|c|}
\hline & Muy satisfactorio & Satisfactorio & Regularmente satisfactorio \\
\hline Grado de satisfacción & 5 & 2 & 0 \\
\hline
\end{tabular}

cartílago auricular y septal, que fueron triturados y morcelizados para posteriormente ser envueltos en una capa doble de material aloplástico (Surgice ${ }^{\circledR}$ ), recomendando una sobrecorrección de $20 \%$ en el aumento del dorso nasal, por la significativa tasa de reabsorción que presentaba este tipo de injerto. Daniel y cols ${ }^{21}$ reportaron un estudio prospectivo de tres grupos. En un grupo se utilizó cartílago cortado envuelto en Surgicel ${ }^{\circledR}$; en el segundo como envoltura se utilizó fascia temporal; y en el tercer grupo no se utilizó envoltura. En el primer grupo todos los pacientes experimentaron una rápida y significativa reabsorción necesitando ser reoperados, no presentándose dicha reabsorción en los otros dos grupos. Tras un análisis histológico, los investigadores atribuyeron esta reabsorción a una reacción a cuerpo extraño secundaria al Surgice ${ }^{\circledR}$.

Recientemente, Tasman $^{22}$ propuso una alternativa para estabilizar los injertos de cartílago cortado sin utilizar una envoltura. Para esto, utilizó tejido tisular adhesivo comercial. El efecto de sellado del adhesivo fija la dispersión de los pequeños injertos y proporciona cierta estabilidad a la estructura permitiendo moldearlo manualmente sin envoltura. Otros autores han estabilizado el cartílago cortado con tejidos adhesivos autólogos obtenidos desde sangre periférica (PRP), creando una estructura relativamente estable que puede ser utilizada en pacientes que necesitan un aumento del dorso nasal ${ }^{23}$.

Nosotros utilizamos injertos de cartílago multifragmentados asociado a pequeños fragmentos de membranas de L-PRF y estabilizados en una matriz de fibrina autóloga, que constituyen un concentrado rico en plaquetas y factores de crecimiento, responsables del gran potencial para la reparación y regeneración de tejidos. Creemos que la matriz de fibrina autóloga con factores de crecimiento 
contenidos en las plaquetas y liberado durante el proceso natural de cicatrización tiene un efecto que mejora ésta misma. El proceso de obtención de LPRF es sencillo y reproducible. Al obtener nuestra fibrina en la segunda centrifugación, ya mezclados los injertos de cartílago y trozos de membranas de L-PRF en un contenedor, se debe añadir a la mezcla antes de 2 minutos ya que alcanza una estructura de gel en aquel tiempo.

El recurso de combinar un injerto de cartílago con una matriz de fibrina autóloga no pretende regenerar células condrales, sino más bien la cohesión del injerto y promover la viabilidad de los condrocitos mediante la acción moduladora de los diferentes factores de crecimiento contenidos en esta estructura, facilitando la formación de tejido conectivo que estabiliza el injerto mixto. Esto establece capilares de neoformación que permiten la difusión de nutrientes a los condrocitos ${ }^{24}$.

Creemos que esta técnica ofrece resultados similares a otras, sin embargo, es técnicamente más fácil y económica. Además, el proceso no incluye substancias químicas. El uso principal que se le dio a este método fue como relleno para aumentar el dorso nasal, sin embargo, también resulta útil en situaciones que se presenten irregularidades $y / 0$ excesiva resección del dorso nasal disminuyendo su altura. Por las características del injerto se puede confeccionar de la longitud y grosor necesario, de modo que se obtenga un dorso totalmente uniforme. Esta técnica ofrece amplias posibilidades en los pacientes secundarios, en los cuales solo obtenemos pequeños trozos de cartílago para injerto y que tengan dorsos irregulares y/o bajos.

El uso de concentrados autólogos ricos en plaquetas en cirugía maxilofacial y ortopédica ha demostrado su eficacia secundaria a la presencia de los mencionados factores de crecimiento pro- moviendo un periodo más corto de cicatrización de heridas ${ }^{25,26}$. Por lo tanto, en un bolsillo de tejidos blandos como el realizado en el dorso nasal en rinoplastías, tendría una mejor cicatrización y la remodelación final del tejido será de mejor cali$\operatorname{dad}^{27-29}$

Creemos que el bajo número de pacientes y el periodo de seguimiento máximo de 24 meses representan limitaciones de nuestro trabajo. No obstante, hasta la fecha los pacientes han mantenido el volumen y la forma inicial sin presentar reabsorción, las cuales resultaron ser estéticamente satisfactorias ante la evaluación de los propios pacientes. Cabe destacar que los resultados menos favorables corresponden a los primeros casos, lo que se atribuye principalmente a la estimación inexacta de la cantidad de injerto requerido y al uso inicial de una jeringa de mayor tamaño como molde. A futuro resulta interesante realizar un análisis histológico de estos injertos con objeto de evaluar el comportamiento y viabilidad de las células mesenquimáticas a largo plazo, y determinar la evolución de este injerto para esclarecer una potencial reabsorción. Esperamos realizar esto en un futuro con mayor cantidad de pacientes.

\section{CONCLUSIÓN}

El injerto mixto representa una nueva alternativa técnicamente fácil, económica, segura y efectiva como relleno de dorso nasal, particularmente útil en secuelas de trauma nasal y rinoplastias secundarias. En ninguno de los casos se presentó reabsorción ni desplazamiento del injerto hasta la fecha. Creemos que el uso de este método también representa una nueva alternativa efectiva para camuflar irregularidades.

\section{BIBLIOGRAFÍA}

1. Strauch B, Erhard Ha, Baum T. Use of irradiated cartilage in rhinoplasty of the non-Caucasian nose. Aesthet Surg J 2004; 24: 324-30.
2. McKinney P, Loomis MG, Wiedrich TA. Reconstruction of the nasal cap with a thin septal graft. Plast Reconstr Surg 1993; 92: 346-51.

3. Sajuadian A, Rubinstein R, Naghshineh N. Current status of grafts and implants in rhinoplasty: part 
I. Autologous grafts. Plast Reconstr Surg 2010; 125: 40e-49e.

4. PARK JH, JIN HR. Use of autologous costal cartilage in Asian rhinoplasty. Plast Reconstr Surg 2012; 130: 1338-48.

5. Kelly MH, Bulstrode NW, Waterhouse N. Versatility of diced cartilage-fascia grafts in dorsal nasal augmentation. Plast Reconstr Surg 2007; 120: 1654-9.

6. Daniel RK, Sajadian A. Secondary rhinoplasty: management of the overresected dorsum. Facial Plast Surg 2012; 28: 417-26.

7. Bielecki T, Dohan Ehrenfest DM. Platelet-rich plasma (PRP) and Platelet-Rich Fibrin (PRF): surgical adjuvants, preparations for in situ regenerative medicine and tools for tissue engineering. Curr Pharm Biotechnol 2012; 13: 1121-30.

8. Dohan Ehrenfest DM, Rasmusson L, Albrektsson T. Classification of platelet concentrates: from pure platelet-rich plasma (P-PRP) to leucocyte- and platelet-rich fibrin (L- PRF). Trends Biotechnol 2009; 27: 158-67.

9. Cieslik-Bielecka A, Choukroun J, Odin G, Dohan EHRENFEST DM. L-PRP/L-PRF in esthetic plastic surgery, regenerative medicine of the skin and chronic wounds. Curr Pharm Biotechnol 2012; 13: $1266-77$.

10. Gibble JW, Ness PM. Fibrin glue: the perfect operative sealant? Transfusion 1990; 30: 741-7.

11. Del Corso M, Vervelle A, Simonpieri A, et al. Current knowledge and perspectives for the use of platelet-rich plasma (PRP) and plateletrich fibrin (PRF) in oral and maxillofacial surgery part 1: Periodontal and dentoalveolar surgery. Curr Pharm Biotechnol 2012; 13: 1207-30.

12. Dohan Ehrenfest DM, Andia I, Zumstein MA, ET AL. Classification of platelet concentrates (Platelet-Rich Plasma-PRP, Platelet-Rich FibrinPRF) for topical and infiltrative use in orthopedic and sports medicine: current consensus, clinical implications and perspectives. Muscles Ligaments Tendons J 2014; 4: 3-9.

13. Rosenthal AR, Egbert PR, Harbury $C$, Hopkins JL, RUBENSTEIN E. Use of platelet- fibrinogen-thrombin mixture to seal experi-mental penetrating corneal wounds. Albrecht Von Graefes Arch Klin Exp Ophthalmol 1978; 207: 111-5.
14. Knighton DR, Ciresi KF, Fiegel VD, Austin LL, ButLER EL. Classification and treatment of chronic nonhealing wounds. Successful treatment with autologous platelet-derived wound healing factors (PDWHF). Ann Surg 1986; 204: 322-30.

15. Whitman DH, Berry RL, Green DM. Platelet gel: an autologous alternative to fibrin glue with applications in oral and maxillofacial surgery. $J$ Oral Maxillofac Surg 1997; 55: 1294-9.

16. Dohan DM, Choukroun J, Diss A, et Al. Plateletrich fibrin (PRF): a second-generation platelet concentrate. Part I: technological concepts and evolution. Oral Surg Oral Med Oral Pathol Oral Radiol Endod 2006; 101: e37-44.

17. Castro-Govea Y, del Campo AF, Chacón-Martínez H, Pérez-Porras S, Vallejo-Estrella R0, CervantesKARDASCH V. A procedure to prevent cephalic rotation of cartilage grafts in the nasal tip. Aesthet Surg J 2009; 29: 98-105.

18. Choukroun J, Diss A, Simonpieri A, et al. Plateletrich fibrin (PRF): a second-generation platelet concentrate. Part V: histologic evaluations of PRF effects on bone allograft maturation in sinus lift. Oral Surg Oral Med Oral Pathol Oral Radiol Endod 2006; 101: 299-303.

19. ERoL 00. The Turkish delight: a pliable graft for rhinoplasty. Plast Reconstr Surg 2000; 105: 2229-41.

20. Elahi MM, Jackson IT, Moreira-Gonzalez A, YaminI D. Nasal augmentation with Surgicel-wrapped diced cartilage: a review of 67 consecutive cases. Plast Reconstr Surg 2003; 111: 1309-18.

21. Daniel RK, Calvert JW. Diced cartilage grafts in rhinoplasty surgery. Plast Reconstr Surg 2004; 113: 2156-171.

22. Tasman AJ, Diener PA, Litschel R. The diced cartilage glue graft for nasal augmentation. Morphometric evidence of longevity. JAMA Facial Plast Surg 2013; 15: 86-94.

23. Bullocks JM, Echo A, Guerra G, Stal S, Yuksel E. A novel autologous scaffold for diced-cartilage grafts in dorsal augmentation rhinoplasty. Aesthetic Plast Surg 2011; 35: 569-79.

24. Kessler MW, Grande DA. Tissue engineering and cartilage. Organogenesis 2008; 4: 28-32.

25. Pal US, Mohammad S, Singh RK, Das S, Singh N, SINGH M. Plateletrich growth factor in oral and maxillofacial surgery. Natl J Maxillofac Surg 2012; 3: 118-23. 
26. Hsu WK, Mishra A, Rodeo SR, et al. Platelet-rich plasma in orthopaedic applications: evidencebased recommendations for treatment. $\mathrm{J} \mathrm{Am}$ Acad Orthop Surg 2013; 21: 739-48.

27. Brenner KA, McConnell MP, Evans GR, Calvert JW. Survival of diced cartilage grafts: an experimental study. Plast Reconstr Surg 2006; 117: 105-15.
28. de Groot PG, Urbanus RT, Roest M. Platelet interaction with the vessel wall. Handbook Exp Pharmacol 2012; 210: 87-110.

29. Chen CW, Corselli M, Péault B, Huard J. Human blood-vesselderived stem cells for tissue repair and regeneration. J Biomed Biotechnol 2012; 2012: 597439 .

Dirección: Alfredo Santamaría Carvallo Servicio Otorrinolaringología y Cirugía de Cabeza y Cuello, Hospital Clínico Regional Guillermo Grant Benavente, Concepción E mail: alsantamariac@gmail.com 